Article

\title{
Isolation and Characterization of Phenolic Compounds from the Leaves of Salix matsudana
}

\author{
Xiang Li ${ }^{1, \dagger}$, Zhi Liu ${ }^{2}$, Xin-feng Zhang ${ }^{3}$, Li-juan Wang ${ }^{1}$, Yi-nan Zheng ${ }^{1}$, Chang-chun Yuan ${ }^{2}$ \\ and Guang-zhi Sun ${ }^{2, *}$
}

${ }^{1}$ College of Chinese Medicinal Materials, Jilin Agricultural University, Changchun, 130118, P.R. China; E-mails: lixiang@bjmu.edu.cn (Xiang Li); ljwang@yahoo.com (Li-juan Wang); zhenyinan@tom.com (Yi-nan Zheng)

${ }^{2}$ Institute of Agricultural Modernization, Jilin Agricultural University, Changchun, 130118, P.R.

China; E-mails: jlndxdhs@126.com; ccyuan@yahoo.com

${ }^{3}$ College of Forestry and Biotechnology, Zhejiang Forestry University, Hangzhou, 311300, P.R. China; E-mail: xhzhang@hotmail.com

$\dagger$ Present address: Agriculture and Agri-Food Canada, Saskatoon Research Center, 107 Science Place, Saskatoon, SK, S7N_0X2, Canada.

* Author to whom correspondence should be addressed; E-mail: gzsun1967@yahoo.com; Tel.: +86-431-8451-0949, Fax: +86-431-8451-0409.

Received: 2 July 2008; in revised form: 31July 2008 / Accepted: 31 July 2008 / Published: 3 August 2008

\begin{abstract}
A bioassay-guided in vitro screen has revealed that a $70 \%$ methanol extract of the leaves of Salix matsudana shows considerable inhibitory activity against cyclooxygenases (COX-1 and COX-2). A subsequent phytochemical study led to the isolation of a new flavonoid, matsudone A (1), together with five known flavonoids - luteolin (2), isoquercitrin (3), 7-methoxyflavone (4), luteolin 7-O-glucoside (5), 4',7-dihydroxyflavone (6) - and two phenolic glycosides, leonuriside A (7) and piceoside (8). Their structures were elucidated on the basis of extensive 1D- and 2D-NMR studies, high resolution ESI mass spectroscopic analyses and comparisons with literature data. The isolated compounds 1-8 were tested for their inhibitory activities against COX-1 and COX-2. Compounds 1, 5 and 6 were found to have potent inhibitory effect on COX-2 and compounds 3-5 exhibited moderate inhibition against COX-1.
\end{abstract}


Keywords: Salix matsudana; matsudone A; flavonoid; cyclooxygenase inhibition.

\section{Introduction}

Salix matsudana Koidz is a small to medium-sized upright spreading tree reaching up to about 30 feet in height and a 15-foot-spread, distributed around the world [1]. In the Traditional Chinese Medical (TCM) literature it was identified as a plant with alexipharmic and antiphlogistic properties, with several pharmacological applications, such as in the treatment of jaundice, hepatitis, rheumatism, and arthritis, as well as eczema [2-3]. Previous phytochemical examinations showed that it produced several kinds of flavonoids, phenolic compounds and diterpene- $\gamma$-lactones [4-6]. As part of our investigations on the chemical constituents and pharmacological activities of Salix species [7-10], a new flavonoid, named matsudone A (1), has been isolated from a 70\% methanol extract of the leaves of Salix matsudana, along with seven known compounds 2-8 (Figure 1). Their structures were elucidated on the basis of high resolution (HR) ESI-MS, ${ }^{1} \mathrm{H}-$ and ${ }^{13} \mathrm{C}-\mathrm{NMR}$, together with $2 \mathrm{D}-\mathrm{NMR}$ spectroscopic analyses. The isolated compounds were tested in a cyclooxygenase (COX-1 and COX-2) bioassay and compounds $\mathbf{1}, 5$ and $\mathbf{6}$ were found to have potent inhibitory effect on COX-2, while compounds 3-5 exhibited moderate inhibition against COX-1.

Figure 1. Flavonoids and phenolic glycosides isolated from the leaves of Salix matsudana.

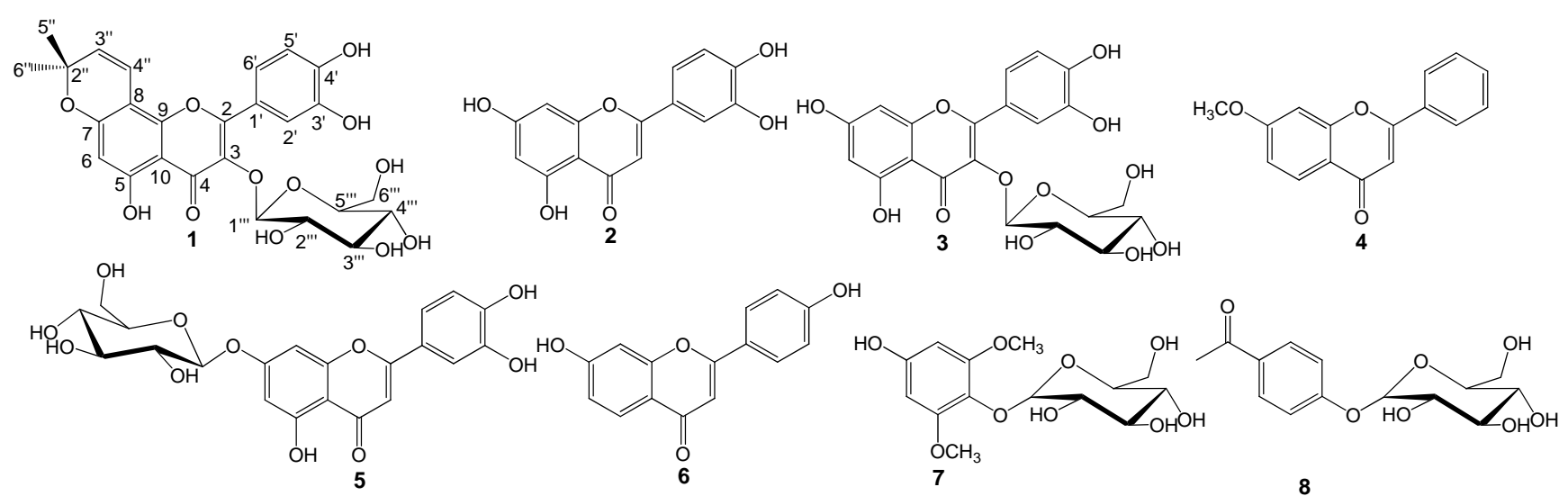

\section{Results and Discussion}

\section{Characterization of compounds $\mathbf{1}-\mathbf{8}$}

Compound 1 was isolated as a pale yellow amorphous powder, and its molecular formula was determined as $\mathrm{C}_{26} \mathrm{H}_{26} \mathrm{O}_{12}$ on the basis of HR-ESI-MS (m/z 531.1517 [M+H] ${ }^{+}$, calcd. 531.1503) and NMR data (Table 1), implying fourteen degrees of unsaturation. The UV (MeOH) bands at 246, 285 (sh), $325 \mathrm{~nm}$ suggested a flavonol skeleton. The ${ }^{13} \mathrm{C}-\mathrm{NMR}$ and DEPT spectra displayed two methyls, one methylene, eleven methines, and twelve quaternary carbons. The phenolic region NMR data of 1 was similar to those of the known compound, 7, 8-(2",2"-dimethylpyrano)-5,3',4'-trihydroxy-3- 
methoxyflavone (Table 1), which had been previously characterized and isolated from the medicinal plant Hypericum Japonicum [11]. The only difference between the two compounds concerned the substitution at C-3, since while the known compound had a methoxyl group at this position, compound 1 was substituted by a sugar group.

Table 1. The NMR data of compound $\mathbf{1}^{\mathrm{a}}$.

\begin{tabular}{|c|c|c|c|c|c|c|c|}
\hline Position & ${ }^{1} \mathrm{H}-\mathrm{NMR}$ & $J(\mathbf{H z})$ & ${ }^{13} \mathrm{C}-\mathrm{NMR}$ & DEPT & COSY & HMBC & ${ }^{13}$ C-NMR $^{\mathrm{b}}$ \\
\hline 2 & - & - & 155.2 & $\mathrm{C}$ & - & - & 155.4 \\
\hline 3 & - & - & 142.7 & $\mathrm{C}$ & - & - & 137.2 \\
\hline 4 & - & - & 178.9 & $\mathrm{C}$ & - & - & 177.5 \\
\hline 5 & - & - & 155.0 & $\mathrm{C}$ & - & - & 154.8 \\
\hline 6 & $6.40, s$ & - & 95.3 & $\mathrm{CH}$ & - & C-8, C-10 & 94.1 \\
\hline 7 & - & - & 160.0 & $\mathrm{C}$ & - & - & 158.0 \\
\hline 8 & - & - & 105.1 & $\mathrm{C}$ & - & - & 103.8 \\
\hline 9 & - & - & 153.9 & $\mathrm{C}$ & - & - & 154.7 \\
\hline 10 & - & - & 105.6 & $\mathrm{C}$ & - & - & 104.6 \\
\hline 1 ' & - & - & 122.1 & $\mathrm{C}$ & - & - & 120.0 \\
\hline $2^{\prime}$ & $7.67, d$ & 1.7 & 117.2 & $\mathrm{CH}$ & H-6' & C-2, C-3', C-4', C-6' & 115.1 \\
\hline $3^{\prime}$ & - & - & 145.3 & $\mathrm{C}$ & - & - & 144.6 \\
\hline $4^{\prime}$ & - & - & 147.6 & $\mathrm{C}$ & - & - & 148.3 \\
\hline 5 ' & $6.95, d$ & 8.5 & 114.7 & $\mathrm{CH}$ & H-6' & $\mathrm{C}-1^{\prime}, \mathrm{C}-3^{\prime}$ & 115.0 \\
\hline $6^{\prime}$ & $7.38, d d$ & $8.5,1.7$ & 120.7 & $\mathrm{CH}$ & H-2', H-6' & C-1', C-2, C-2', C-4' & 120.0 \\
\hline $2 ”$ & - & - & 78.1 & $\mathrm{C}$ & - & - & 77.4 \\
\hline $3 ”$ & $5.57, d$ & 10.0 & 125.7 & $\mathrm{CH}$ & H-4" & C-5"', C-6"', C-7, C-8 & 128.5 \\
\hline $4 ”$ & $6.56, d$ & 10.0 & 116.3 & $\mathrm{CH}$ & H-3" & C-5"', C-6"', C-7, C-9 & 113.9 \\
\hline $5 ”$ & $1.47, s$ & - & 27.3 & $\mathrm{CH}_{3}$ & - & C-2"', C-3"' & 27.2 \\
\hline 6” & $1.47, s$ & - & 27.3 & $\mathrm{CH}_{3}$ & - & C-2"', C-3"' & 27.2 \\
\hline 1, & $5.15, d$ & 7.6 & 102.2 & $\mathrm{CH}$ & H-2"' & C-3, C-3"' & - \\
\hline $2, "$ & $3.18, m$ & - & 72.7 & $\mathrm{CH}$ & - & - & - \\
\hline $3, "$, & $3.2-3.5, m$ & - & 77.5 & $\mathrm{CH}$ & - & - & - \\
\hline $4, "$ & $3.2-3.5, m$ & - & 70.6 & $\mathrm{CH}$ & - & - & - \\
\hline $5, "$, & $3.2-3.5, m$ & - & 76.9 & $\mathrm{CH}$ & - & - & - \\
\hline 6", & $3.65, d$ & 11.5 & 62.1 & $\mathrm{CH}_{2}$ & H-6"' & C-4"', C-5"' & - \\
\hline -OMe & - & - & - & - & - & - & 59.1 \\
\hline
\end{tabular}

${ }^{a}$ Compound 1 was measured in DMSO- $d_{6}$ and chemical shifts are expressed in ppm; ${ }^{\mathrm{b}}{ }^{13} \mathrm{C}-\mathrm{NMR}$ data (in DMSO- $\left.d_{6}\right)$ of 7,8-(2"', 2"'-dimethylpyrano)-5, 3', 4'-trihydroxy-3-methoxyflavone [11].

This finding was supported by ${ }^{13} \mathrm{C}-\mathrm{NMR}$ data which showed a group of sugar signals at $\delta_{\mathrm{C}} 102.2(\mathrm{~d}$, C-1"'), $\delta_{\mathrm{C}} 77.5$ (d, C-3"'), $\delta_{\mathrm{C}} 76.9$ (d, C-5"'), $\delta_{\mathrm{C}} 72.7$ (d, C-2'"), $\delta_{\mathrm{C}} 70.6$ (d, C-4'") and $\delta_{\mathrm{C}} 62.1$ (t, C-6"'). This sugar moiety was identified as glucose on the basis of an acid hydrolysis reaction, whose reaction product showed same $R_{f}$ on the TLC plate as an authentic glucose reference. The coupling constant 
$J_{\mathrm{H} 1 \text { '", H2"' }}(7.6 \mathrm{~Hz})$ of 1 indicated a $\beta$-glucose. The absolute configuration of the $\beta$-glucose was further determined to be $\beta$-D-glucose by chiral GC analysis. Meanwhile, H-1"' $(\delta$ 5.15) showed HMBC correlation with $\delta_{\mathrm{C}-3} 142.7$ (Figure 2), confirming that the glucose moiety was connected to the C-3 position. Thus, compound 1 was determined to be 7,8-(2", 2"-dimethylpyrano)-5, 3', 4'-trihydroxyflavone-3-O- $\beta$-D-glucoside, to which we have given the name matsudone $\mathrm{A}$.

Figure 2. Partial HMBC and COSY correlations of compound 1.

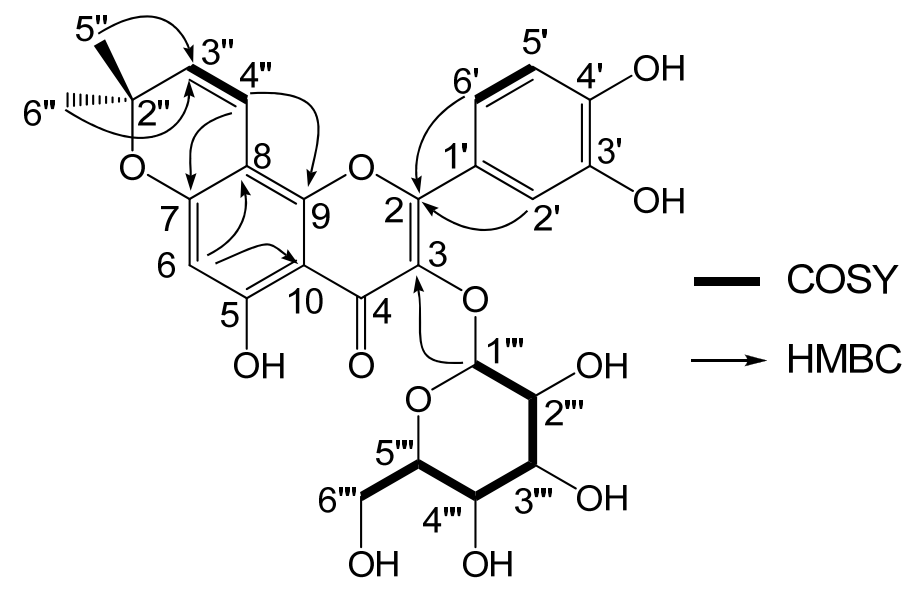

The known compounds 2-8 were identified on the basis of comparison of their NMR data with that found in the literature [12-18]. The sugar moieties of compounds 3, 5, 7 and 8 were determined by the acid hydrolysis method, as mentioned in the Experimental section. The results showed that all the above compounds contained the same sugar moiety, $\beta$-D-glucose.

Figure 3. Proposed biosynthetic pathway of matsudone A (1) and isolated flavonoids 2, 3 and 5.

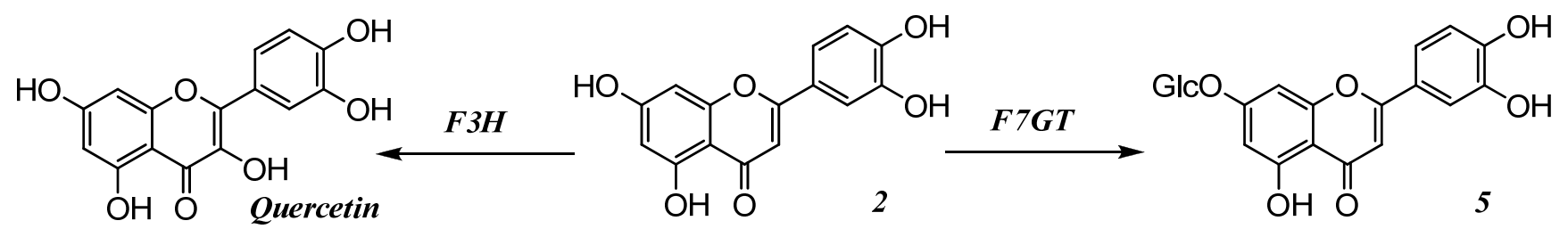

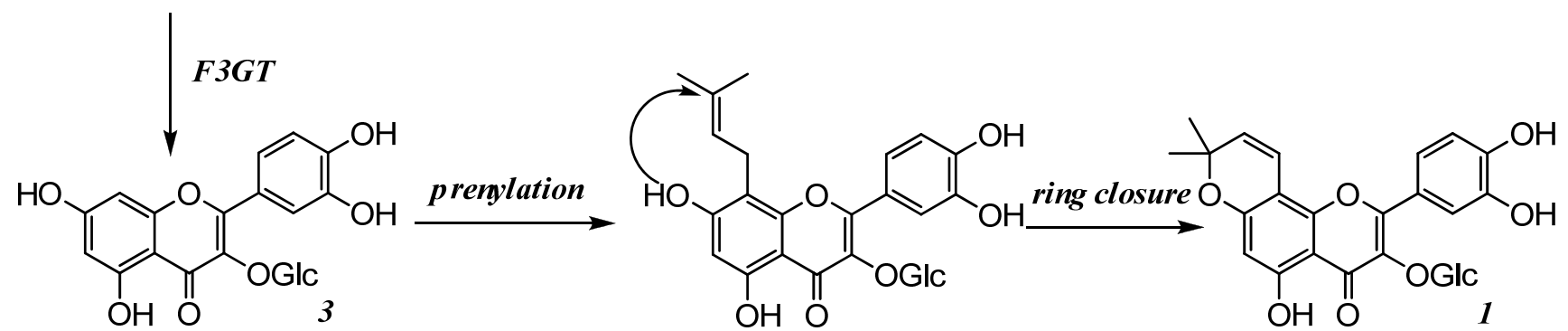

Flavonoids are among the best-characterized plant secondary metabolites in terms of chemistry, mechanism of coloration, biochemistry, genetics and molecular biology [19]. In the present study, we isolated several flavonoids, 1-6, for which the biosynthetic pathway shown in Figure 3 may be proposed. 
Briefly, luteolin (2) could afford the common flavonoids quercetin and luteolin 7-O-glucoside (5), via reactions catalyzed by the enzymes flavonoid 3-hydroxylase $(F 3 H)$ and flavonoid 7-glucosyltransferase $(F 7 G T)$, respectively. Quercetin could be further catalyzed by flavonoid 3-glucosyltransferase (F3GT) to form isoquercetrin (3), and compound 1 could be formed after prenylation and ring closure. Although to our knowledge no publications have reported flavonoid pathway related genes in Salix matsudana, our results strongly suggested that the speculated enzymes (F3H, F7GT, F3GT and prenyltransferase) exist in this spp.

\section{Bioactivity Results}

The isolated compounds were tested for their inhibitory activities against cyclooxygenase (COX-1 and COX-2, Table 2). Compounds 1, 5 and $\mathbf{6}$ were found to have potent inhibitory effect on COX-2, with $\mathrm{IC}_{50}$ values $(\mu \mathrm{M})$ of $27.3,39.1$ and 58.8, respectively; compounds 3-5 exhibited moderate inhibition against $\mathrm{COX}-1$, with $\mathrm{IC}_{50}$ values $(\mu \mathrm{M})$ of 102.7 , 99.1 and 92.3 , respectively. Interestingly, the new isolated matsudone A (1) showed considerable COX-2 inhibition activity $\left(\mathrm{IC}_{50}=27.3 \mu \mathrm{M}\right)$ compared to the positive control aspirin $\left(\mathrm{IC}_{50}=19.0 \mu \mathrm{M}\right)$, making it a good candidate for further consideration as an anti-inflammatory prodrug.

Table 2. $\mathrm{IC}_{50}$ data of isolated compounds as inhibitors of COX-1 and COX-2 $2^{\mathrm{a}}$.

\begin{tabular}{cccccccccc}
\hline$\#$ & $\mathbf{1}$ & $\mathbf{2}$ & $\mathbf{3}$ & $\mathbf{4}$ & $\mathbf{5}$ & $\mathbf{6}$ & $\mathbf{7}$ & $\mathbf{8}$ & Aspirin \\
\hline IC $_{\mathbf{5 0}}$ of COX-1 ( $\left.\boldsymbol{\mu M}\right)$ & 153.1 & I.A. $^{\mathrm{b}}$ & 102.7 & 99.1 & 92.3 & I.A. & 195.4 & 216.9 & 21.7 \\
IC $_{\mathbf{5 0}}$ of COX-2 $(\boldsymbol{\mu M})$ & 27.3 & I.A. & I.A. & 169.0 & 39.1 & 58.8 & 199.7 & I.A. & 19.0 \\
\hline \multicolumn{7}{c}{ Values are means of three determinations. ${ }^{\mathrm{b}}$ I.A.: Inactive. }
\end{tabular}

\section{Conclusions}

In summary, a novel flavonoid, matsudone A (1), has been isolated from leaves of Salix matsudana, together with seven known compounds, 2-8. The isolated compounds 1-8 were tested for their inhibitory activities against COX-1 and COX-2. Compounds 1, 5 and $\mathbf{6}$ were found to have potent inhibitory effects on COX-2 and compounds 3-5 exhibited moderate inhibition against COX-1.

\section{Experimental}

\section{General}

The ${ }^{1} \mathrm{H}$ - and ${ }^{13} \mathrm{C}-\mathrm{NMR}$ spectra were measured on a Bruker Avance DRX 500 NMR spectrometer, using TMS as an internal standard. Chemical shifts $(\delta)$ are expressed in parts per million (ppm), with the coupling constants $(J)$ reported in Hertz $(\mathrm{Hz})$. The ESI-MS spectra were recorded on a triple quadrupole mass spectrometer Quattro (VG Biotech, Altrincham, England) and the HRESI-MS spectra on a Bruker FT-ICRMS spectrometer. Column chromatographies were carried out with silica gel $60 \mathrm{M}$ (200-300 mesh), Lichrospher RP-18 (20 $\mu \mathrm{m})$ and Sephadex LH-20 (Pharmacia); TLC was performed 
on silica gel plates (Macherey-Nagel, SilG/UV $\mathrm{UV}_{254}, 0.20 \mathrm{~mm}$ ), with spots detected by $\mathrm{UV}_{254}$ and anisaldehyde $/ \mathrm{H}_{2} \mathrm{SO}_{4}(10 \%)$. HPLC was performed on an Agilent 1100 instrument.

\section{Chemicals and reagents}

COX-1 and COX-2 were purchased from Cayman Chemical (Michigan, US). The ${ }^{14} \mathrm{C}-$ labeled arachidonic acid $(>200 \mu \mathrm{Ci}, \mathrm{NEN})$ was purchased from New England Nuclear Co. (Boston, US). Other chemicals and reagents were purchased from the Chinese Chemical Group (Beijing, P.R. China).

\section{Extraction and isolation}

Leaves of Salix matsudana was collected on the campus of Jilin Agricultural University (JLAU) and identified by one of the authors, Prof. Yi-nan Zheng. A voucher specimen (HLY-06-01) has been deposited in the Laboratory of Medicinal Chemistry, JLAU. Crude powdered Salix matsudana leaves $(6.5 \mathrm{~kg})$ were extracted with $70 \% \mathrm{MeOH}(20 \mathrm{~L})$ at room temperature for $48 \mathrm{~h}$, and the extract concentrated to give a dark brown residue $(850 \mathrm{~g})$. This residue was blended with silica gel and directly subjected to chromatography on a silica column, eluted with gradient mixture of $\mathrm{CHCl}_{3}$ $\mathrm{MeOH}-\mathrm{H}_{2} \mathrm{O}\left(9: 1,5: 1\right.$ and 1:1), to yield five fractions $\left(\mathrm{F}_{\mathrm{A}}-\mathrm{F}_{\mathrm{E}}\right)$. The fourth fraction, $\mathrm{F}_{\mathrm{D}}$, was subjected to silica gel chromatography, eluted with $\mathrm{CHCl}_{3}-\mathrm{MeOH}-\mathrm{H}_{2} \mathrm{O}=7: 2: 0.1$, then further purified by semipreparative HPLC (gradient elution of $5 \%$ aqueous $\mathrm{MeOH}$ to $100 \% \mathrm{MeOH}$ ) to afford compounds 1 (7 $\mathrm{mg})$ and $7(12 \mathrm{mg})$. The fifth fraction, $\mathrm{F}_{\mathrm{E}}$, was subjected to ODS RP-18 column chromatography eluted with $80 \% \mathrm{MeOH}$ to afford compounds $3(106 \mathrm{mg}), 6(12 \mathrm{mg})$ and $2(49 \mathrm{mg})$. The third fraction, $\mathrm{F}_{\mathrm{C}}$, was subjected to further chromatography on a Sephadex LH-20 column, with $70 \% \mathrm{MeOH}$ elution, to afford compounds 4 (7 mg), 5 (135 mg) and 8 (26 mg).

\section{Acid hydrolysis of compounds 1, 3, 5, 7 and $\mathbf{8}$}

Compounds 1, 3, 5, 7 and 8 (each $2.0 \mathrm{mg}$ ) were refluxed with $6 \mathrm{~N} \mathrm{HCl}(5 \mathrm{~mL})$ at $100{ }^{\circ} \mathrm{C}$ for $2 \mathrm{~h}$. Each mixture was extracted with $\mathrm{CHCl}_{3}$ to afford the corresponding aglycone, and the aqueous layer was neutralized with $\mathrm{Na}_{2} \mathrm{CO}_{3}$ and filtered. The aqueous layer was dried under vacuum and the residue was re-dissolved in $\mathrm{H}_{2} \mathrm{O}$ for sugar analysis by TLC with $n$ - $\mathrm{BuOH}-\mathrm{HOAc}-\mathrm{H}_{2} \mathrm{O}$ (4:1:2) as the solvent. The sample spots were detected by spraying aniline hydrogen phthalate reagent $(100 \mathrm{~mL} n-\mathrm{BuOH}$ saturated by $\mathrm{H}_{2} \mathrm{O}, 0.96 \mathrm{~g}$ aniline and $1.66 \mathrm{~g}$ phthalic acid) and heating. Authentic samples of glucose, xylose and rhamnose were used as standards. The absolute configuration of the glucose detected was further determined by chiral GC analysis using a SatoChrom GC and a $0.25 \mathrm{~mm}$ x $25 \mathrm{~m}$ Hydrodexb-6TBDM chiral capillary column (Macherey-Nagel, Germany). $\beta$-D-glucose was used as an authentic GC standard. The aqueous layer residues mentioned above were re-suspended in dichloromethane $(1 \mathrm{~mL})$, and trifluoroacetic anhydride $(50 \mu \mathrm{L})$ was added. The mixtures were allowed to react at room temperature overnight and dried under a stream of nitrogen at room temperature. The sugar derivatives were separated using the following temperature program: inlet temperature was set at $240{ }^{\circ} \mathrm{C}$, with hydrogen carrier gas and a 1/20 split, using nitrogen makeup gas. Column temperatures started at 120 ${ }^{\circ} \mathrm{C}$, ramped to $220{ }^{\circ} \mathrm{C}$ at $50{ }^{\circ} \mathrm{C} \mathrm{min}-1$ and were maintained for $12 \mathrm{~min}$. 
Matsudone A (1). Obtained as a pale yellow amorphous powder; $[\alpha]_{\mathrm{D}}^{20}-59.1(c 0.5, \mathrm{MeOH}) ; \mathrm{mp} 349.1-$ $349.9{ }^{\circ} \mathrm{C}$; UV (MeOH) $\lambda_{\max }: 246,285(\mathrm{sh}), 325 \mathrm{~nm}$; HRESI-MS [+]: $m / z=531.1517[\mathrm{M}+\mathrm{H}]^{+}$(calcd. for $\left.\mathrm{C}_{26} \mathrm{H}_{27} \mathrm{O}_{12}, 531.1503\right) ;{ }^{1} \mathrm{H}$ and ${ }^{13} \mathrm{C}$-NMR data, see Table 1.

Effect on Cyclooxygenase-1 and -2

The effect of the tested compounds on cyclooxygenase- 1 and -2 (COX-1 and -2) was determined by measuring $\mathrm{PGE}_{2}$ production. Generally, the reaction mixtures were prepared in Tris- $\mathrm{HCl}$ buffer $(\mathrm{pH}$ $8.0)$, containing hematin $(1.5 \mu \mathrm{M})$, glutathione $(300 \mu \mathrm{M})$, epinephrine $(300 \mu \mathrm{M})$, enzyme (COX-1 or $\mathrm{COX}-2,50 \mu \mathrm{L})$ and various concentrations of isolated compounds. $1-{ }^{14} \mathrm{C}$ Arachidonic acid $(10 \mu \mathrm{L})$ was added to start the reaction. The mixture was incubated for $30 \mathrm{~min}$ at $37^{\circ} \mathrm{C}$, then, the reaction was terminated by adding the reaction mixture $(20 \mu \mathrm{L})$ to $30 \mu \mathrm{M}$ indomethacin $(200 \mu \mathrm{L})$. Arachidonic acid and its radio-labeled metabolites were separated and determined by reversed-phase HPLC using a Berthold radioactivity monitor. Inhibition refers to reduction of $\mathrm{PGE}_{2}$ formation, in comparison to a blank run without inhibitor. The results are means of three independent experiments. Aspirin was used as a positive control.

\section{Acknowledgements}

We are grateful to Professor Dr. Zhiwei Deng (Analytical Center, Beijing Normal University) for measuring the NMR spectra. This work was partially supported by a grant from JATCM (Jilin Academy of Traditional Chinese Medicine and Materia Medica).

\section{References and Notes}

1. Kuzovkina, Y.; Quigley M. Willows beyond wetlands: uses of Salix L. species for environmental projects. Water Air Soil Poll. 2005, 162, 183-204.

2. Li, S. Ben Cao Gang Mu (in Chinese); The People's Health Press: Bei Jing, P.R. China, 1982; pp. 2039-2040.

3. Jiangsu New Medical College. Dictionary of Traditional Chinese Medicine (in Chinese); Jiangsu Technology Press: Nan Jing, China, 1977; pp. 1123-1124.

4. Zheng, Y.; Zhang, J.; Han, L.; Sekiya, K.; Kimura, Y.; Okuda, H. Effects of compounds in leaves of Salix matsudana on arachidonic acid metabolism. Yakugaku Zasshi 2005, 125, 1005-1008.

5. Xu, C.; Zheng, Y.; Yang, X.; Li, X.; Li, X.; Chen, Q. Raddeanalin, a new flavonoid glycoside from the leaves of Salix raddeana Laksh. J. Asian Nat. Prod. Res. 2007, 9, 415-419.

6. Wang, J.; Zheng, S.; Shen, T.; Shen, X.; Li, Y. Two New Acyclic Diterpene- $\gamma$-lactones from the Leaves of Salix matsudana. Chin. Chem. Lett. 2002, 5, 432-435.

7. Zhang, J.; Zheng, Y.; Han, L. Studies on chemical constituents of leaves of Salix matsudana Koidz and their influence on lipolysis. Zhong Guo Zhong Yao Za Zhi 2000, 25, 538-541.

8. Zhang, J.; Zheng, Y.; Han, L. Isolation of resisting thrombus and arteriosclerosis compounds in leaves of Salix matsudana. Zhong Yao Cai 1999, 22, 131-133. 
9. Han, L.; Sumiyoshi, M.; Zhang, J.; Liu, M.; Zhang, X.; Zheng, Y.; Okuda, H.; Kimura, Y. Antiobesity action of Salix matsudana leaves (Part 1). Anti-obesity action by polyphenols of Salix matsudana in high fat-diet treated rodent animals. Phytother. Res. 2003, 17, 1188-1194.

10. Han, L.; Sumiyoshi, M.; Zheng, Y.; Okuda, H.; Kimura, Y. Anti-obesity action of Salix matsudana leaves (Part 2). Isolation of anti-obesity effectors from polyphenol fractions of Salix matsudana. Phytother. Res. 2003, 17, 1195-1198.

11. Wu, Q.; Wang, S.; Du, L.; Zhang, S.; Yang, J.; Xiao, P. Chromone glycosides and flavonoids from Hypericum Japonicum. Phytochemistry 1998, 49, 1417-1420.

12. Peters, N.; Frost, J.; Long, S.; A plant flavone, luteolin, induces expression of Rhizobium meliloti dodulation gene. Science 1986, 233, 977-980.

13. Pakudina, Z.; Leontev, V.; Kamaev, F.; Sadykov, A. Structure and PMR spectra of isoquercitrin and hirsutrin. Chem. Nat. Comp. 1973, 5, 572-574.

14. Ambrozin, A.; Vieira, P.; Fernandes, J.; Silva, M.; Albuquerque, S. Trypanocidal activity of Meliaceae and Rutaceae plant extracts. Mem. Inst. Oswaldo Cruz 2004, 99, 227-231.

15. Chiruvella, K.; Mohammed, A.; Dampuri, G.; Ghanta, R.; Raghavan, S. Phytochemical and antimicrobial studies of methyl angolensate and luteolin-7-O-glucoside isolated from callus cultures of Soymida febrifuga. Int. J. Biomed. Sci. 2007, 4, 269-278.

16. Tsetsegmaa, S., Batsurén, D., Dungerdorzh, D.; Batirov, E.; Malikov, V. A chemical study of plants of the Mongolian flora the flavonoids of two species of Oxytropis. Chem. Nat. Comp. 1993, 28, 628-629.

17. Otsuka, H.; Takeuchi, M.; Inoshiri, S.; Sato, T.; Yamasaki, K. Phenolic compounds from Coix jachryma-jobi var. Ma-yuen. Phytochemistry 1989, 28, 883-886.

18. Mitrocots, D.; Skaltsounis, A.; Mitaku, S.; Harvala, C.; Tillequin, F. Flavonoid and terpene glycosides from European Ebenus species. Biochem. Syst. Ecol. 1999, 27, 305-307.

19. Tanaka, Y.; Sasaki, N.; Ohmiya, A. Biosynthesis of plant pigments: anthocyanins, betalains and carotenoids. Plant J. 2008, 54, 733-749.

Sample Availability: Samples are available from the co-author Xiang Li (lixiang@bjmu.edu.cn).

(C) 2008 by the authors; licensee Molecular Diversity Preservation International, Basel, Switzerland. This article is an open-access article distributed under the terms and conditions of the Creative Commons Attribution license (http://creativecommons.org/licenses/by/3.0/). 